\title{
A Monte Carlo investigation of four nonparametric multiple-comparison tests for $\mathrm{k}$ independent groups
}

\author{
EDWARD L. WIKE and JAMES D. CHURCH \\ The University of Kansas, Lawrence, Kansas 66045
}

\begin{abstract}
A Monte Carlo study of four nonparametric multiple-comparison tests ( $t)$, the Wilcoxon, Steel, Nemenyi, and Ryan tests, was done to determine their Type I error rates when overall Kruskal-Wallis $\mathrm{H}$ tests were and were not significant. A total of 13,000 simulated experiments, $\mathrm{N}$, were performed in a design with $\mathrm{k}=3,5,7$, and 9 groups and $\mathrm{n}=7,10$, and 15 replicates per group at $\alpha=.05$ and and $\alpha=.01$. It was found that: (1) most of the variance in the error rates was accounted for by $\mathrm{k}, \mathrm{t}$, and $\mathrm{k} \times \mathrm{t}$; (2) when the Wilcoxon test was used for pairwise comparisons after a significant $\mathrm{H}$ test, it was the best of the four procedures. The latter finding is concordant with recent studies of parametric multiple-comparison tests.
\end{abstract}

Despite compelling evidence for the robustness of fixed-effects analysis of variance (ANOVA) tests (Glass, Peckham, \& Sanders, 1972), some investtigators still routinely use nonparametric methods or use them when departures from the assumptions for ANOVA are marked. In the case of $k$ independent groups, the Kruskal-Wallis $\mathrm{H}$ test of the hypothesis of simultaneous equality of the $\mathrm{k}$ distributions is usually employed as an overall nonparametric test. Subsequent pairwise comparisons among groups are then typically carried out by Wilcoxon's ranksum tests of the hypothesis of pairwise equality of the distributions. ${ }^{1}$ However, alternative multiple comparison tests exist (Hollander \& Wolfe, 1973; Miller, 1966) which incorporate adjustments for the number of comparisons being made. The primary aim of the present Monte Carlo study was to compare the Type I error performance of three such tests with Wilcoxon's test.

Earlier Monte Carlo investigations of parametric multiple-comparison tests (e.g., Petrinovich \& Hardyck, 1969) determined error rates without respect to the significance of the overall $\mathrm{F}$ test. More recently, Bernhardson (1975) and Carmer and Swanson (1973) have asked a more realistic question from a research worker's point of view: How do various multiple-comparison tests perform when the overall $\mathrm{F}$ test is significant? This new question has produced some surprising and important results. Carmer and Swanson found overall Type I comparisonwise error rates and experimentwise error rates close to the intended 5\% for Fisher's least significant difference (l.s.d.) tests when they were performed after significant $\mathrm{F}$ tests. Furthermore, the 1.s.d. test showed excellent power, that is, the efficient detection of differences in the nonnull case. And these findings generalized across a wide range of groups $(\mathrm{k}=5,10$, and 20$)$ with small numbers of replicates ( $\mathrm{n}=3,4,6$, and 8 ). Bernhardson compared the l.s.d., HSD, Newman-Keuls, Duncan multiple-range, and Scheffé tests over all simulated experiments and in those experiments in which the overall $F$ test was significant. In the null case, at $\alpha=.05$, over $k=2$, $4,6,8$, and 10 and $n=15$, the l.s.d. test's comparisonwise error rates (over all experiments) and experimentwise error rates (when $F$ was significant) were both very close to the $5 \%$ level. Bernhardson reduced his data to six different error rates. As these error rates are applicable to the present study, they are described below.

A significant comparison refers to a rejection of the null hypothesis of equality of a pair of means by a single paired-comparison test at a given significance level. An experiment for which $\mathrm{F}$ is significant refers to a rejection of the null hypothesis of simultaneous equality of $\mathrm{k}$ means at a given significance level. The six error rates are defined as follows: (1) The comparisonwise error rate $\left(\alpha_{1}\right)$ is the total number of significant comparisons divided by the total number of comparisons; that is, the product of the total number of experiments, $\mathrm{N}$, and the number of comparisons per experiment, $k(k-1) / 2$. Two other comparisonwise error rates, $\alpha_{A}$, and $\alpha_{B}$, are obtained using pairs from experiments in which $F$ is significant. (2) $\alpha_{A}$ is the number of significant comparisons when $\mathrm{F}$ is significant divided by the total number of comparisons, $\mathrm{Nk}(\mathrm{k}-1) / 2$. (3) $\alpha_{\mathrm{B}}$ is the number of significant comparisons when $F$ is significant divided by the product of the number of experiments in which $F$ is significant and $k(k-1) / 2$. Paralleling these comparisonwise error rates are three experimentwise error rates. (4) The overall experimentwise error rate $\left(\alpha_{2}\right)$ is the total number of experiments with one or more significant comparisons 
divided by the total number of experiments, N. (5) $\alpha_{\mathrm{C}}$ is the number of experiments with one or more significant comparisons in which $\mathrm{F}$ is significant divided by the total number of experiments, N. (6) $\alpha_{\mathrm{D}}$ is the number of experiments with one or more significant comparisons when $\mathrm{F}$ is significant divided by the number of experiments in which $\mathrm{F}$ is significant.

The aims of our study can now be stated more specifically: (1) To evaluate the Type I error performance of the Kruskal-Wallis $\mathrm{H}$ test for small samples; (2) to determine the six Type I error rates for Wilcoxon's rank-sum test (Hollander \& Wolfe, 1973). Steel's test (Miller, 1966), a "conservative", version of Nemenyi's test (Hollander \& Wolfe, 1973; Miller, 1966), and Ryan's test (Ryan, 1960) for small samples $(\mathrm{k}=3,5,7$, and 9 and $\mathrm{n}=7$, 10 , and 15); (3) to assess the relative effects of the three independent factors (tests, number of groups, and number of replicates) that were included in the Monte Carlo study upon the six kinds of error rates; and (4) to comment upon the utility of the different Type I error rates and offer a recommendation as to the optimal nonparametric multiple-comparison test for $\mathrm{k}$ independent groups for use following a significant $\mathrm{H}$ test.

\section{METHOD}

\section{Monte Carlo Procedures}

For every $\mathrm{k}, \mathrm{n}$ combination, the ranks $1,2, \ldots, \mathrm{kn}$ were randomly assorted into $\mathrm{k}$ sets of $\mathrm{n}^{2}$. A total of 1,000 runs were made on a Honeywell $66 / 60$ computer for every $k, n$ combination except for the 7,15 combination, in which 2,000 runs were performed. Thus, a total of 13,000 simulated experiments, $N$, were carried out. For each experiment a Kruskal-Wallis $\mathrm{H}$ test and the four multiple-comparison tests were done at significance levels of $\alpha=.05$ and $\alpha=.01$. Counts were obtained for: (1) the number of experiments in which $\mathrm{H}$ was significant; (2) the total number of significant comparisons; (3) the number of significant comparisons in which $\mathrm{H}$ was significant; (4) the total number of experiments with one or more significant comparison; and (5) the number of experiments with one or more significant comparison and for which $\mathrm{H}$ was significant. It should be noted that since the ranks $1,2, \ldots, k n$ were assorted randomly for every simulated experiment, each significant comparison is a Type I error. The study yielded no direct information regarding the nonnull case, that is, the occurrence of Type II errors and the power of the various tests. A further limitation was that only the equal-n case was studied throughout.

\footnotetext{
Multiple-Comparison Tests

The four multiple-comparison tests were selected because of their procedural diversity and the availability of tables of test distributions. The Nemenyi test utilizes the rank sums from the $\mathrm{H}$ test in performing pairwise comparisons; for the other three tests, the rank sums for the two groups being compared are the result of re-ranking the combined integers for the two groups. The Ryan test, a variant of the Tukey B test, involves layerwise comparisons with different probability values being required for significance, depending upon the number of steps separating the groups after they have been ordered in accord with their original rank sums. On the other hand, the Wilcoxon, Steel, and Nemenyi tests each have a single critical value for assessing the significance of all pairwise comparisons from each $\mathrm{k}, \mathrm{n}$ combination at a given $\alpha$ value.
}

The test statistic for the Wilcoxon rank-sums test is $\mathrm{T}^{\prime}$, the larger rank sum for the two groups being compared. The critical values for $T^{\prime}$ were obtained from the Wilcoxon, Katti, and Wilcox tables (1963). The $T^{\prime}$ values depend only upon $n$, the number of replicates, in the two groups under comparison and are invariant with respect to $k$, the number of groups. The statistic for Steel's test is also $T^{\prime}$. The critical values for $T^{\prime}$ for Steel's test, which are dependent upon both $\mathrm{k}$ and $\mathrm{n}$, may be found in Miller (1966, Table 7). At $k=9$ and $n=7$, there is no $T^{\prime}$ value that is significant for $\alpha=.05$. The same problem occurs at $\mathrm{k}=5,7$, and 9 and $\mathrm{n}=7$ for $\alpha=.01$. For all of these combinations, a $T^{\prime}=77$ was required. This is the maximum rank sum which is possible for two groups with $n=7$. The effect of using this arbitrary critical value should be to increase slightly the observed incidence of Type I errors for Steel's test at these k,n combinations. The test statistic for Nemenyi's test is: $\mathbf{H}^{\prime}=$ $\left(R_{\cdot u}-R_{\cdot v}\right)^{2} /[k n(k n+1) / 12](2 / n)$, in which $R_{\cdot u}$ and $R_{\cdot v}$ are the average ranks based upon the rank sums in the original ranking. Because the $\mathrm{k}, \mathrm{n}$ combinations exceeded the 3,5 limits of the Kruskal-Wallis table, critical values of $\mathbf{H}^{\prime}$ were obtained from the $\chi^{2}$ distribution with $\mathrm{k}-1$ degrees of freedom. Finally, the test statistic for Ryan's test is: $Z=\left(R_{u}^{\prime}-R_{\cdot}^{\prime}\right) /[2 n(2 n+1) / 12]^{1 / 2}$ $(2 / n)^{1 / 2}$, in which $R^{\prime}{ }_{u}$ and $R^{\prime}$, are the average ranks following re-ranking. For the two extreme groups, the two-tailed $P$ value for $\mathrm{Z}$ had to be equal to or larger than $2 \alpha / \mathrm{k}(\mathrm{k}-1)$ for significance. For groups one step less extreme, the required $P$ value was $2 \alpha / \mathrm{k}(\mathrm{k}-2)$, and so on. It may be noted again that all simulations involved equal ns and the formulas are for this case only.

\section{RESULTS}

Prior to considering the error rates for the multiplecomparison tests, it is essential to examine the performance of the Kruskal-Wallis $\mathrm{H}$ test under these relatively small sample conditions. At the .05 level across all $12 \mathrm{k}, \mathrm{n}$ combinations, the null hypothesis was rejected in $4.46 \%$ of the experiments; at the .01 level the obtained value was $.75 \%$. Thus, although the $\mathrm{H}$ test was slightly conservative with respect to Type I errors, the observed rejection frequencies were not deemed sufficiently discrepant to jeopardize the investigation of the multiple-comparison tests when the overall $\mathrm{H}$ test was significant.

The six error rates for the four tests (t) at every $\mathrm{k}, \mathrm{n}$ combination were calculated for $\alpha=.05$ and $\alpha=.01 .^{3}$ Elsewhere (Wike \& Church, 1977) we have argued that statistical tests, ANOVAs, and percents of accounted-for-variance, should be applied to Monte Carlo data in addition to the traditional methods of inspecting tables and graphs. Because the present Monte Carlo study had a balanced factorial design, a $4(\mathrm{k})$ by $3(\mathrm{n})$ by $4(\mathrm{t})$ plan, the six error rates were subjected to a series of ANOVAs that revealed that 58 out of 72 possible effects were significant. To cope with this large array of significant effects and to estimate their magnitudes, percentages of accounted-for variance in the six error rates were calculated using Hays' (1963) formulas. Examination of the $\omega^{2}$ values revealed that: (1) three effects, $\mathrm{k}, \mathrm{t}$, and $\mathrm{k} X \mathrm{t}$, when combined, accounted for most of the variance ranging from $46 \%$ for the $\alpha_{\mathrm{C}}$ variance at $\alpha=.01$ to $98 \%$ for $\alpha_{1}$ at $\alpha=.05$; 
Table 1

Error Rates for the Four Tests for Different Numbers of Groups (k) at $\alpha=.05$

\begin{tabular}{|c|c|c|c|c|c|c|c|c|c|c|c|c|}
\hline \multirow[b]{4}{*}{ Test } & \multicolumn{12}{|c|}{ Comparisonwise Error Rates } \\
\hline & \multicolumn{4}{|c|}{$\alpha_{1}$} & \multicolumn{4}{|c|}{$\alpha_{\mathbf{A}}$} & \multicolumn{4}{|c|}{$\alpha_{\mathbf{B}}$} \\
\hline & \multicolumn{4}{|c|}{$\mathrm{k}$} & \multicolumn{4}{|c|}{$\mathrm{k}$} & \multicolumn{4}{|c|}{ k } \\
\hline & 3 & .5 & 7 & 9 & 3 & 5 & 7 & 9 & 3 & 5 & 7 & 9 \\
\hline Wilcoxon & .055 & .053 & .052 & .052 & .024 & .016 & .010 & .010 & .512 & .326 & .260 & .221 \\
\hline Steel & .015 & .005 & .002 & .001 & .013 & .003 & .001 & .001 & .288 & .072 & .025 & .015 \\
\hline Nemenyi & .013 & .002 & .000 & .000 & .013 & .002 & .000 & .000 & .274 & .036 & .006 & .001 \\
\hline Ryan & .030 & .012 & .006 & .004 & .016 & .004 & .001 & .001 & .352 & .087 & .030 & .013 \\
\hline
\end{tabular}

Experimentwise Error Rates

\begin{tabular}{|c|c|c|c|c|c|c|c|c|c|c|c|c|}
\hline \multirow[b]{3}{*}{ Test } & \multicolumn{4}{|c|}{$\alpha_{2}$} & \multicolumn{4}{|c|}{$\alpha_{\mathbf{C}}$} & \multicolumn{4}{|c|}{$\alpha_{\mathbf{D}}$} \\
\hline & \multicolumn{4}{|c|}{ k } & \multicolumn{4}{|c|}{$\mathrm{k}$} & \multicolumn{4}{|c|}{ k } \\
\hline & 3 & 5 & 7 & 9 & 3 & 5 & 7 & 9 & 3 & 5 & 7 & 9 \\
\hline Wilcoxon & .138 & .296 & .478 & .602 & .048 & .048 & .039 & .045 & 1.000 & 1.000 & 1.000 & 1.000 \\
\hline Steel & .040 & .037 & .026 & .029 & .036 & .027 & .016 & .020 & .751 & .560 & .406 & .418 \\
\hline Nemenyi & .036 & .016 & .004 & .002 & .036 & .016 & .004 & .002 & .747 & .333 & .124 & .042 \\
\hline Ryan & .077 & .105 & .118 & .132 & .039 & .028 & .017 & .017 & .818 & .592 & .429 & .365 \\
\hline
\end{tabular}

(2) the main effects of $\mathrm{k}$ and $\mathrm{t}$ were always larger than $\mathrm{k} \mathrm{X} \mathrm{t}$; and (3) the effects of $\mathrm{n}$ were small by comparison but tended to be larger at $\alpha=.01$.

The statistical analyses of the six error rates suggest that the data are best summarized by $\mathrm{k}$ by $\mathrm{t}$ tables in which the ns are combined. Table 1 shows the six error rates for the .05 level. The comparisonwise error rates, $\alpha_{1}, \alpha_{A}$, and $\alpha_{B}$, are displayed in the upper panel and the experimentwise error rates, $\alpha_{2}, \alpha_{C}$, and $\alpha_{\mathrm{D}}$, in the lower panel. The $\alpha_{1}$ error rate for the Wilcoxon test at $k=3$ was .055 . Stated another way, $5.5 \%$ of the comparisons were significant out of the total number of comparisons. It is also apparent that the Wilcoxon $\alpha_{1}$ error rate did not change with $\mathrm{k}$. The $\alpha_{1}$ error rates for the other three tests fell below $5 \%$ at $\mathrm{k}=3$ and decreased rapidly as $\mathrm{k}$ increased. Considering the numbers of significant comparisons from experiments in which $\mathrm{H}$ was significant, the Wilcoxon test had the highest error rates whether the number of significant comparisons was divided by the total number of comparisons $\left(\alpha_{A}\right)$ or by the number of comparisons in experiments in which $\mathrm{H}$ was significant $\left(\alpha_{\mathrm{B}}\right)$. Both of these error rates decreased as $\mathrm{k}$ increased, but the changes were less rapid for the Wilcoxon test than for the other tests.

In the experimentwise error rates, the Wilcoxon and Ryan tests had the highest overall $\left(\alpha_{2}\right)$ rates, that is the number of experiments with at least one significant comparison divided by the total number of experiments. In addition, their $\alpha_{2}$ rates increased as $\mathrm{k}$ became larger, while the lower $\alpha_{2}$ rates for the Steel and Nemenyi tests decreased as $\mathbf{k}$ became larger. Considering the number of experiments with at least one significant comparison and for which $\mathrm{H}$ was significant, the Wilcoxon test had the highest error rates whether the number of experiments $\left(\alpha_{C}\right)$ or the number of experiments for which $\mathrm{H}$ was significant $\left(\alpha_{\mathrm{D}}\right)$ was the referent. It is also noteworthy that the Wilcoxon $\alpha_{C}$ and $\alpha_{D}$ error rates were stable across $\mathrm{k}$, while they dropped for the other three tests. The $\alpha_{C}$ rate of $4.5 \%$, on the average, for the Wilcoxon test means that the number of experiments with $\mathrm{H}$ significant and at least one significant comparison was $4.5 \%$ of all experiments. The $\alpha_{D}$ rate for the Wilcoxon test of $100 \%$ means that in every experiment in which $\mathrm{H}$ was significant at least one significant comparison was found.

In Table 2 the six error rates for the .01 level are displayed. These error rates showed the same patterns of result with respect to $k$ and $t$ that were found at $\alpha=.05$.

\section{DISCUSSION}

The finding that Type I error rates for the Kruskal-Wallis $\mathrm{H}$ test tended to be slightly conservative at both $\alpha=.05$ and $\alpha=.01$ is in accord with a previous Monte Carlo study by Gabriel and Lachenbruch (1969). They reported that the degree of bias in the $\mathrm{H}$ test was related to $\mathrm{n}$, but in the present study the observed Type I error rates appeared to be independent of both $k$ and $n$. It should be noted, however, that the range of $k, n$ combinations was much more extensive in the Gabriel and Lachenbruch study.

The comparisonwise and experimentwise error rates for the four nonparametric multiple-comparisons tests manifested striking similarities with the previous findings of Bernhardson (1975) and Carmer and Swanson (1973) on parametric multiplecomparison procedures. For example, Bernhardson observed $\alpha_{1}$ and $\alpha_{C}$ error rates of close to $5 \%$ and an $\alpha_{D}$ error rate of $100 \%$ for the l.s.d. test - the same error rates were obtained in our study with Wilcoxon's test. In general, Bernhardson found that the other multiple-comparisons tests in this study (the HSD, Newman-Keuls, Duncan, and Scheffé tests) were too conservative. Similarly, Tables 1 and 2 reveal that the Steel, Nemenyi, and Ryan tests resulted in too few significant comparisons. It might be argued that the application of the Wilcoxon test is risky because of its excessively high overall experimentwise error rates $\left(\alpha_{2}\right)$ ranging 
Table 2

Error Rates for the Four Tests for Different Numbers of Groups (k) at $\alpha=.01$

\begin{tabular}{|c|c|c|c|c|c|c|c|c|c|c|c|c|}
\hline \multirow[b]{4}{*}{ Test } & \multicolumn{12}{|c|}{ Comparisonwise Error Rates } \\
\hline & \multicolumn{4}{|c|}{$\alpha_{1}$} & \multicolumn{4}{|c|}{$\alpha_{\mathbf{A}}$} & \multicolumn{4}{|c|}{$\alpha_{\mathbf{B}}$} \\
\hline & \multicolumn{4}{|c|}{$\mathrm{k}$} & \multicolumn{4}{|c|}{$\mathbf{k}$} & \multicolumn{4}{|c|}{$\mathbf{k}$} \\
\hline & 3 & 5 & 7 & 9 & 3 & 5 & 7 & 9 & 3 & 5 & 7 & 9 \\
\hline Wilcoxon & .011 & .011 & .010 & .010 & .003 & .002 & .001 & .001 & .476 & .242 & .164 & .134 \\
\hline Steel & .002 & .001 & .000 & .000 & .001 & .000 & .000 & .000 & .250 & .061 & .021 & .015 \\
\hline Nemenyi & .001 & .000 & .000 & .000 & .002 & .000 & .000 & .000 & .228 & .022 & .004 & .000 \\
\hline Ryan & .014 & .008 & .005 & .003 & .002 & .000 & .000 & .000 & .287 & .045 & .013 & .007 \\
\hline
\end{tabular}

Experimentwise Error Rates

\begin{tabular}{|c|c|c|c|c|c|c|c|c|c|c|c|c|}
\hline \multirow[b]{3}{*}{ Test } & \multicolumn{4}{|c|}{$\alpha_{2}$} & \multicolumn{4}{|c|}{$\alpha_{\mathbf{C}}$} & \multicolumn{4}{|c|}{$\alpha_{\mathbf{D}}$} \\
\hline & \multicolumn{4}{|c|}{ k } & \multicolumn{4}{|c|}{ k } & \multicolumn{4}{|c|}{ k } \\
\hline & 3 & 5 & 7 & 9 & 3 & 5 & 7 & 9 & 3 & 5 & 7 & 9 \\
\hline Wilcoxon & .031 & .080 & .139 & .208 & .007 & .008 & .008 & .008 & 1.000 & 1.000 & 1.000 & 1.000 \\
\hline Steel & .005 & .007 & .005 & .008 & .004 & .004 & .002 & .003 & .722 & .512 & .297 & .422 \\
\hline Nemenyi & .004 & .002 & .001 & .000 & .004 & .002 & .001 & .000 & .656 & .216 & .083 & .060 \\
\hline Ryan & .039 & .074 & .098 & .113 & .005 & .004 & .002 & .002 & .750 & .420 & .241 & .222 \\
\hline
\end{tabular}

from about $14 \%$ for $k=3$ to about $60 \%$ for $k=9$. If experimenters did not employ overall tests like the Kruskal-Wallis $\mathrm{H}$ test prior to doing multiple-comparison tests, then we would agree with this argument. However, most experimenters, quite correctly, do overall tests first. Then the critical question is: How do the various multiple-comparison tests perform when the overall test is significant? The error rates that provide answers to this question are $\alpha_{\mathrm{A}}, \alpha_{\mathrm{B}}, \alpha_{\mathrm{C}}$, and $\alpha_{\mathrm{D}}$, rather than $\alpha_{1}$ and $\alpha_{2}$. In the case of $k$ independent groups, our study demonstrates clearly that the Wilcoxon test is the optimal procedure for performing pairwise comparisons after a significant KruskalWallis $\mathrm{H}$. Because the Kruskal-Wallis $\mathrm{H}$ tends to be somewhat conservative and the $F$ test is remarkably robust under equal n conditions, we do not advocate the use of the Kruskal-Wallis $\mathrm{H}$ test unless the departures from the ANOVA assumptions are clearly marked. In any event, our study points to the Wilcoxon test as the recommended test for pairwise multiple comparisons after obtaining a significant $\mathrm{H}$ value. If these tactics are followed, the investigator will falsely reject the null hypothesis with respect to one or more comparisons in about 5 experiments $\left(\alpha_{\mathrm{C}}=.045\right)$ out of 100 at $\alpha=.05$, and he will always find at least one significant comparison for every experiment in which he rejects the overall null hypothesis $\left(\alpha_{\mathrm{D}}=1\right)$. What about the power of the Wilcoxon test? Although only Type I errors were studied here, when $\mathrm{k}>2$ the Wilcoxon test requires smaller critical values for significance than the values for the other three multiplecomparison procedures. Accordingly, when $k>2$, under nonnull conditions, the Wilcoxon Type II error rates must be the smallest and its power the greatest of the four tests.

\section{REFERENCES}

Bernhardson, C. S. Type I error rates when multiple comparison procedures follow a significant $\mathrm{F}$ test of ANOVA. Biometrics, 1975, 31, 229-232.

Carmer, S. G., \& Swanson, M. R. An evaluation of ten pairwise multiple comparison procedures by Monte Carlo methods. Journal of the American Statistical Association, 1973, 68, 66-74.

GABRIEL, K. R., \& LACHENbRUCh, P. A. Non-parametric ANOVA in small samples: A Monte Carlo study of the adequacy of the asymptotic approximation. Biometrics, 1969, 25, 539-596.

Glass, G. V., Peckham, P. D., \& Sanders, J. R. Consequences of failure to meet assumptions underlying the fixed-effects analysis of variance and covariance. Review of Educational Research, 1972, 42, 237-288.

Hays, W. L. Statistics for psychologists. New York: Holt, Rinehart, \& Winston, 1963.

Hollander, M., \& Wolfe, D. A. Nonparametric statistical methods. New York: Wiley, 1973.

Miller, R. G., JR. Simultaneous statistical inference. New York: McGraw-Hill, 1966.

Petrinovich, L. F., \& Hardyck, C. D. Error rates for multiple comparison methods: Some eridence concerning the frequency of erroneous conclusions. Psychological Bulletin, 1969, 71, 43-54.

Ryan, T. A. Significance tests for multiple comparisons of proportions, variances, and other statistics. Psychological Bulletin, 1960, 57, 318-328.

Wike, E. L., \& Church, J. D. Analysis of variance methods for the design and analysis of Monte Carlo statistical studies. Bulletin of the Psychonomic Society, 1977, 10, 131-133.

Wilcoxon, F., Katti, S. K., \& Wilcox, R. A. Critical values and probability levels for the Wilcoxon rank sum test and the signed rank test. American Cyanamid Company \& Florida State University, 1963.

\section{NOTES}

1. Actually, rejections of the null hypothesis for the KruskalWallis and Wilcoxon tests are regarded as indicants of location differences as a consequence of treatment effects.

2. We wish to thank Dr. Charles Neuringer for suggesting an efficient algorithm for randomly assorting ranks.

3. As indicated above, an adjusted $T^{\prime}$ critical value was used in four $\mathrm{k}, \mathrm{n}$ combinations for Steel's test, in which there were no critical values. Since the observed error rates in these instances were comparable to the error rates for the other $\mathrm{k}, \mathrm{n}$ combinations, they were treated as data.

(Received for publication September 22, 1977.) 